\title{
The Impact of Ly49-NK Cell-Dependent Recognition of MCMV Infection on Innate and Adaptive Immune Responses
}

\author{
Michal Pyzik,, 2,3 Eve-Marie Gendron-Pontbriand, ${ }^{2,3}$ and Silvia M. Vidal, 2,3,4 \\ ${ }^{1}$ Department of Human Genetics, McGill University, Montreal, QC, Canada H3G OB1 \\ ${ }^{2}$ Centre for the Study of Host Resistance, McGill University, Montreal, QC, Canada H3G1A4 \\ ${ }^{3}$ McGill Complex Traits Group, McGill University, Montreal, QC, Canada H3GOB1 \\ ${ }^{4}$ Department of Microbiology and Immunology, McGill University, Montreal, QC, Canada H3A 2B4 \\ Correspondence should be addressed to Silvia M. Vidal, silvia.vidal@mcgill.ca
}

Received 26 January 2011; Accepted 15 March 2011

Academic Editor: John E. Coligan

Copyright () 2011 Michal Pyzik et al. This is an open access article distributed under the Creative Commons Attribution License, which permits unrestricted use, distribution, and reproduction in any medium, provided the original work is properly cited.

Clinical and experimental data indicate that a subset of innate lymphocytes, natural killer (NK) cells, plays a crucial role in the response against herpesviruses, especially cytomegaloviruses (CMV). Indeed, in mice, NK cells, due to the expression of germline encoded Ly49 receptors, possess multiple mechanisms to recognize CMV infection. Classically, this results in NK cell activation and the destruction of the infected cells. More recently, however, this unique host-pathogen interaction has permitted the discovery of novel aspects of NK cell biology, implicating them in the regulation of adaptive immune responses as well as in the development of immunological memory. Here, we will concisely review the newly acquired evidence pertaining to NK cell Ly49-dependent recognition of MCMV-infected cell and the ensuing NK cell regulatory responses.

If one way be better than another, that you may be sure is nature's way.

Aristotle

\section{Introduction}

Natural killer (NK) cells constitute a unique effector lymphocyte lineage, classically defined as part of the innate immune system. Their importance is well characterized in the context of health, under normal physiological conditions. For instance, NK cells are involved in tumor surveillance, given their natural cytotoxicity against neoplasms. In pregnancy, NK cells account for $70 \%$ of decidual leukocytes; they are thought to mediate trophoblast invasion into the uterine lining and the modification of maternal spiral arteries [1]. In addition to their implication in these crucial components of homeostasis and normal development, NK cells are well known for their considerable role in defense against infection.

Infection with a wide variety of pathogens can be limited by the action of NK cells, specifically intracellular microorganisms. Indeed, NK cells play a substantial role in the initial control of both bacterial (e.g., Listeria monocytogenes) and parasitic (e.g., Plasmodium species) spread [2]. Yet, in the context of infection, the hallmark of NK cells remains their potent antiviral activity. NK cells participate in the clearance of many different viruses (human immunodeficiency virus, coxsackievirus, influenza or poxviruses, etc.), but their contribution is indispensable with regards to infection with members of the Herpesviridae family (herpes simplex virus (HHV-1/2), varicella zoster virus (HHV-3), and cytomegalovirus (HHV-5)) [2]. Plentiful evidence supports this, namely, the cases of two young patients suffering from numerous and recurrent herpesviral infections due to their nonfunctional NK cells $[3,4]$. In mice, NK cell depletion and adoptive transfer have long been known to increase, respectively, susceptibility and resistance to mouse CMV (MCMV).

The antiviral activity of NK cells relies on the various effector functions induced following their activation. On the 
one hand, NK cells secrete several cytokines, such as IFN$\gamma$, MIP1- $\alpha / \beta$, RANTES, IL-10, activation-induced T-cellderived chemokine-related cytokine (ATAC, lymphotactin), and others. On the other hand, NK cells display strong cytotoxicity. Indeed, they can kill virus-infected cells without prior activation, and they do so via a number of mechanisms, namely exocytosis of perforin-granzymes granules and signaling through members of the TNF death receptor family [5]. Yet, cytokine production and cytotoxicity must necessarily be preceded by the recognition of infected cells by NK lymphocytes, a phenomenon that has mostly been studied using a mouse model of infection.

CMVs are prototypical $\beta$-subgroup members of the Herpesviridae family. Given the nonredundant role played by NK cells in resolving infections with these viruses, it is not surprising that CMVs would be perfect candidates for the study of NK cell responses. Unfortunately, the strict species specificity of CMVs precludes experimental infection of mice with human CMV (HCMV). Yet, MCMV shares many features with its human counterpart, including genome structure and disease manifestations [6]. Both are natural pathogens of their respective host and have coevolved with them for eons. Moreover, both viruses have developed varied and analogous immune-evasion mechanisms that heavily implicate NK cells [7]. Therefore, early MCMV infection has become an established model to study NK cells and, more specifically, their impressive ability to distinguish self from nonself through their germ-line encoded receptors. In addition, at later times post-infection, this model reveled the unforeseen involvement of NK cells in the adaptive immune responses.

NK cells discriminate between infected and healthy cells using an extensive panel of cell surface receptors, both activating and inhibitory. Among the various receptor families involved in this process, Ly49 receptors have proven themselves to be particularly important for MCMV detection by murine NK cells. These polygenic and polymorphic receptors are clustered at the Natural Killer Cell Complex (NKC) on mouse chromosome 6 [8]. They are stochastically expressed as disulfide-linked homodimers primarily on the surface of NK cells, but also on subsets of monocytes, macrophages, dendritic cells (DCs), and T cells [9]. In terms of ligand specificity, inhibitory Ly49 receptors recognize selfMHC class I molecules (MHCI, also called $\mathrm{H}-2$ in mice), whereas their activating counterparts can bind to various protein determinants of infection.

Ly49 receptors are structurally classified as type II transmembrane, C-type lectin-like proteins. Their extracellular domain is comprised of a flexible stalk and a Natural Killer Domain (NKD), which provides ligand binding specificity and is structurally conserved among all members of the Ly49 family. Yet, activating and inhibitory receptors differ with regards to their intracellular domains. Indeed, inhibitory Ly49 receptors possess an immunoreceptor tyrosine-based inhibition motif (ITIM) within their intracellular domain. Conversely, activating Ly49 receptors lack this ITIM motif; instead, a positively charged arginine residue in their transmembrane domain interacts with the DAP12/DAP10 adaptor proteins, which bears an immunoreceptor tyrosinebased activation motif (ITAM).

During infection, Ly49 receptor triggering leads to the initiation of a signaling cascade, the result of which is either inhibition or activation of the NK cell. In the case of both activating and inhibitory receptors, the first step of this cascade is the phosphorylation of the tyrosine residue contained in their respective ITAM or ITIM, most likely by a Src family kinase [5]. This phosphorylation recruits either SHIP-1, SHP-1, or SHP-2 in the case of inhibitory receptors, or Syk, ZAP-70 and $\mathrm{PI}(3) \mathrm{K}$ or Grb2 for activating receptors. In both cases, numerous downstream effectors are involved. The end result of inhibitory receptor triggering is the dephosphorylation of ITAMs linked to activating $\mathrm{NK}$ receptors and the prevention of $\mathrm{Ca}^{2+}$ influx, degranulation, cytokine production, and NK cell proliferation. In opposition, activating Ly49 receptor engagement induces the reorganization of the actin cytoskeleton to enable cell polarization and the release of cytolytic granules, as well as the transcription of many cytokine and chemokine genes.

Of note, inhibition is by far the most common signal received by NK cells over the course of their lifetime. Moreover, during NK cell development, binding of inhibitory Ly49 receptors to self-MHCI molecules renders them functional, that is to say capable of cytokine secretion (e.g., IFN- $\gamma$ ) and cytotoxic killing [10]. This model, called NK cell education or licensing, postulates that the stronger the inhibitory Ly49-self MHCI interactions are, the more competent the NK cell will emerge. The immune response which follows MCMV infection relies on the interplay of soluble factors and numerous cell types, among them NK cells. In the early innate response, Ly49 receptors are critical for NK cell activation, which in turn controls viral proliferation, due to their ability to recognize various viral and self-ligands. Nevertheless, initial NK cell proliferation is Ly49-independent and nonselective, while the subsequent preferential NK cell proliferation is driven by activating Ly 49 receptors. At later stages of the infection, NK cell secreted cytokines and apt virus control allows them to regulate the adaptive response. Most surprisingly, Ly49-mediated activation of NK cells also allows the generation of NK cells with several characteristics seen in memory T cells (Figure 2(a)).

All of these aspects of MCMV-Ly49-driven NK cell responses will be examined in this paper. Most importantly, evidence will be presented to highlight the previously unanticipated sophistication of the NK cell response against infection, redefining the established concepts of innate and adaptive immunity.

\section{Immediate Nonspecific NK Cells Response to MCMV Infection}

From its initial peripheral sites of entry, MCMV spreads to the lymph nodes and via the bloodstream to the spleen, and the liver, infecting a broad spectrum of cell types. Macrophages, hepatocytes, and reticular fibroblasts are some of its primary targets $[11,12]$. At $6-8$ hours after infection (p.i.), lymphotoxin $\alpha / \beta$ expressed on the surface of $B$ cells 
interacts with its cognate receptor on splenic stromal cells, triggering type I interferon (IFN- $\alpha / \beta$ ) secretion by the latter $[13,14]$. Within 24 hours after the initial infection, before the end of the first round of viral division, this first wave of type I IFN secretion wanes. Subsequently, a second round of viral infection occurs, this time in many more organs, making the infection systemic.

Over the course of MCMV infection, several molecular viral byproducts are generated due to the lytic nature of the pathogen. Plasmacytoid DCs (pDCs) engulf infected cell debris and recognize them through their endosomal pattern recognition receptors (PRRs), that is, TLR3, TLR7 and TLR9; this recognition induces a second, much stronger wave of type I IFN secretion ( $~ 36$ hours p.i) as well as the production of other proinflammatory cytokines (IL-12, IL-6, IL- $1 \beta$, TNF- $\alpha$, etc.) [15-17]. In parallel, the presence of MCMV particles in the cytoplasm of pDCs can lead to viral recognition by the NOD-like and RIG-I-like helicase sensors resulting in secretion of more pro-inflammatory cytokines; these molecules probably also contribute to pDCindependent secretion of type I IFN [18-20].

Conventional DCs (cDCs) are one of the many cell types that are initially infected by MCMV. In response to infection, they secrete IL-12, IL-18, IL-15 (trans-presented), and type I IFN all of which are required for NK cell activation. Indeed, different NK cell effector functions require different cytokine inputs for their initiation: while the IFN- $\alpha \beta /$ STAT1 pathway is necessary for cytotoxicity and initial proliferation, NK cell IFN- $\gamma$ production during the first 2 days following infection necessitates IL-18 or IL-12/STAT4 (Figure 2(a)i) [21-23]. Moreover, the IFN- $\gamma$ secreted by infected cDCs, along with macrophage-secreted MIP1- $\alpha$, are critical for the control of viral loads in the liver and lungs [24]. IFN- $\gamma$ is also important for perforin-dependent control of viral replication in the spleen [25]. Once activated, NK cells start to eliminate infected cDCs and TNF- $\alpha$-secreting macrophages, thus reducing the immunopathology caused by this cytokine [26]. In short, the initial NK cell response against MCMV is mediated by a cytokine storm; it is rapid, nonspecific, and global, involving NK cells with diverse Ly49 repertoires. Yet, it does not effectively control viral spread.

Indeed, despite the high levels of antiviral cytokines present in the serum, MCMV replication proceeds unhindered. This is true for all inbred strains at day 1.5 after infection. Yet, by day 3 p.i., mouse strains can be segregated according to their NK cell-dependent control of MCMV [27]. Most strains are susceptible to the virus, while a select few are resistant. These strains include C57BL/6 (B6) and $\mathrm{MA} / \mathrm{MyJ}$ mice, whose resistance depends on their strainspecific activating Ly49 receptor, as well as PWK/Pas and NZW/LacJ mice, whose resistance is not yet characterized [27-29].

\section{Specific Recognition of MCMV-Infected Cells}

3.1. Ly49H/m157 Axis. MCMV resistance in B6 mice depends on the presence of the activating Ly49H receptor on the surface of their NK cells [30-32]. In this mouse strain, approximately $50 \%$ of NK cells express the receptor. Ly $49 \mathrm{H}$ binds directly to m157, a MCMV-encoded glycoprotein with structural homology to host MHCI molecules; unlike the latter, however, m157 is a GPI-anchored protein, does not associate with $\beta_{2}$-microglobulin, or present peptides at the surface of the cell [33-35]. During the early phase of the infection, Ly49H-m157 ligation initiates an activating signaling cascade, resulting in clonal proliferation of Ly $49 \mathrm{H}^{+}$ NK cells, killing of infected cells, and secretion of cytokines (IFN- $\gamma$, MIP1- $\alpha$, TNF- $\alpha$, etc.), all of which result in the reduction of viral loads (Figure 1(a)).

The importance of this receptor-ligand pair in the control of MCMV infection was demonstrated by numerous studies. In the case of the virus, resistant mice became susceptible upon infection with a m157-deletant MCMV ( $\Delta m 157)$ (Figure 1(b)) [36]. As for the host, deletion of Ly49H gene or deletion or loss of function mutation of the DAP12 gene were shown to render mice susceptible to MCMV infection, as revealed by high viral titers [37-39]. Conversely, transgenesis of Ly49H into initially susceptible strains was found to make them resistant to MCMV [40].

Once the interaction between Ly49H and m157 was established as an essential component of MCMV resistance in B6 mice, the nature of this interaction was investigated. Structural analysis, molecular modeling, and targeted mutations of both $\mathrm{m} 157$ and $\mathrm{Ly} 49 \mathrm{H}$ have shown the importance of the receptor homodimerization domain. Indeed, Ly49H receptors carrying mutations in this region display reduced $\mathrm{m} 157$ recognition, while mutations in the putative ligandbinding regions had limited impact on this same interaction [41]. In the same vein, mutation of buried residues required for correct $\mathrm{m} 157$ folding $(\alpha 0: \alpha 2$ helix interactions) altered the ability of the viral molecule to bind $\mathrm{Ly} 49 \mathrm{H}$, while mutations in the exposed and scattered residues across the entire m157 structure did not [42]. Furthermore, posttranslational modifications can also affect Ly49H-m157 binding. Indeed, given the four putative $\mathrm{N}$-glycosylation motifs (NXT/S) of m157, a variety of glycosylated isoforms were found to be expressed on the surface of MCMV-infected cells. Although these isoforms are also recognized by Ly49H, their binding stability and half-life are increased as compared to the unglycosylated m157 [43]. These results reveal a quite tolerant recognition of $\mathrm{m} 157$ by $\mathrm{Ly} 49 \mathrm{H}$ where several amino acid substitutions are necessary in order to disrupt m157Ly49H interactions. Such a molecular arrangement could allow Ly $49 \mathrm{H}$ to recognize variable $\mathrm{m} 157$ molecules, emerging as the MCMV attempts to escape immune recognition.

From an evolutionary standpoint, capture of the $\mathrm{H}$ 2 gene by MCMV is thought to have initiated a cascade of events leading to the emergence of the Ly49H-m157 interaction. Originally, this capture could have allowed the virus to exploit a MHCI homologue capable of triggering inhibitory Ly49 receptors. Since the Ly49 locus evolves rapidly, any inhibitory receptor could have been converted to an activating one, including those with binding specificity for m157 [44, 45]. In support of this, m157 can be bound by the inhibitory Ly49I receptor from 129/J mice; unfortunately, the involvement of Ly49I in susceptibility to MCMV infection could not be assessed due to a defect in 


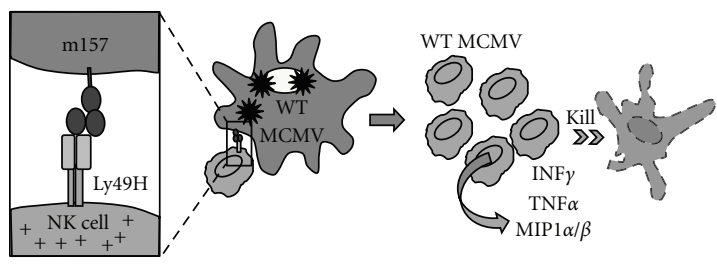

(a)

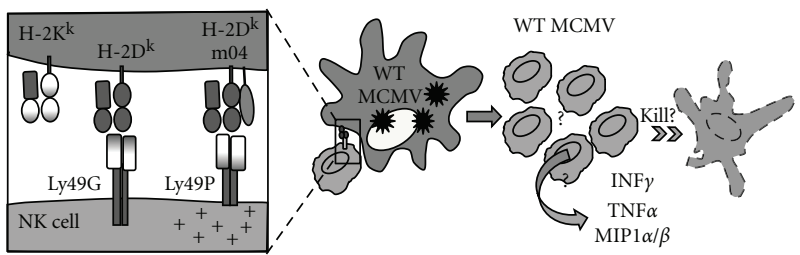

(c)

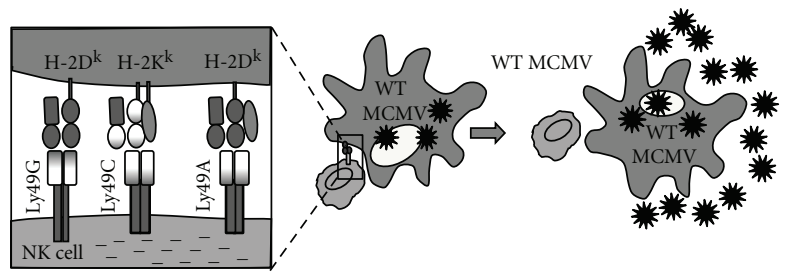

(e)

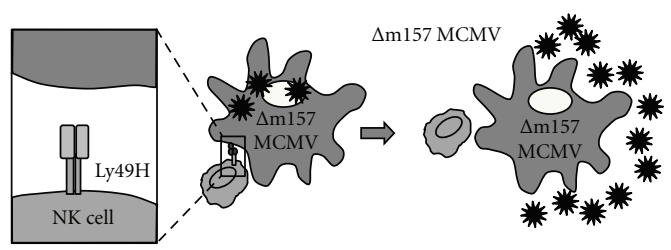

(b)

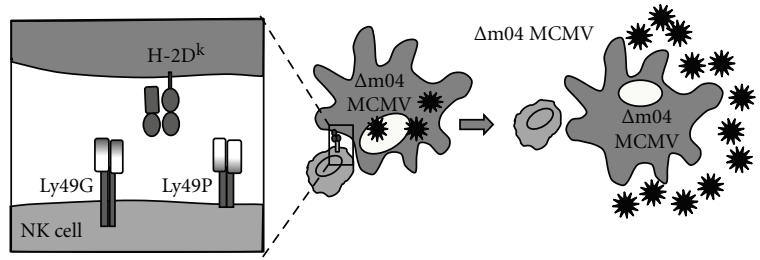

(d)

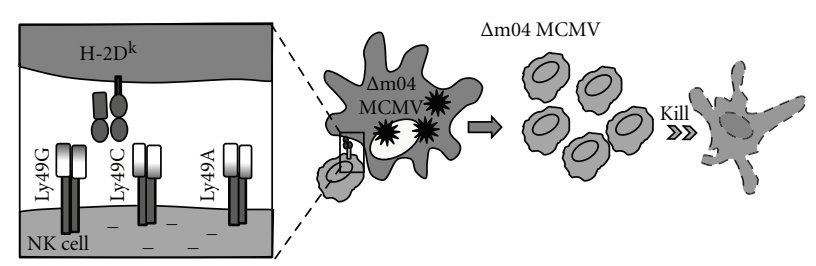

(f)

FIGURE 1: Multiple modes of Ly49-NK cell-dependent recognition of MCMV-infected cell. NK cell-dependent detection of MCMV infection occurs through different Ly49 receptors and viral proteins. (a, b) Ly49H-m157, operating in C57BL/6 mice; (c, d) Ly49P-m04:H-2Dk or Ly49G-H-2D ${ }^{k}$, operating in MA/MyJ mice; (e, f) Inhibitory Ly49-H-2:m04, operating in BALB mice. (a, c) Triggering of the activating Ly49 receptors by their viral ligand, $\mathrm{m} 157$, or by their virally modified self-ligands, $m 04: \mathrm{H}-2 \mathrm{D}^{\mathrm{k}}$, leads to NK cell cytotoxicity, proliferation, and secretion of cytokines. (b, d) The absence of these ligands prevents NK cells from recognizing and responding to infected cells. (e) However, triggering of inhibitory Ly49 receptors by virally modified self-ligands results in NK cell unresponsiveness during the infection. (f) NK cell responsiveness is restored upon infection with a deletant virus lacking the protein required for modifying self-ligands. WT MCMV: Wild-type MCMV, $\triangle$ m157: MCMV lacking m157, $\Delta$ m04: MCMV lacking m04, (+): activation, (-): inhibition.

NK cell signaling within this strain [33]. Recently, additional inhibitory Ly49 receptors, namely, Ly49C from the B6, $\mathrm{BALB} / \mathrm{c}$, and NZB strains, have been shown to interact with the m157 obtained from wild-derived MCMV isolates [46]. Interestingly, Ly49C-specific recognition of nonlaboratory strain-MCMV-infected cells did not seem to interfere with Ly49H-mediated NK cell activation and the ensuing antiviral response in $\mathrm{B} 6$ mice. The dominant $\mathrm{Ly} 49 \mathrm{H}$ recognition of $\mathrm{m} 157$ is supported by the fact that sequential passage of wildtype (WT) MCMV in B6 mice leads to the emergence of "NK cell-escape" m157 variants, which evade Ly49H-mediated NK cell recognition [47]. Nevertheless, the m157 proteins of MCMV strains isolated from wild mice are highly variable, indicating that outside of laboratory settings, both inhibitory and activating Ly 49 receptors could be targeted by the virus. In any case, the fact that $\mathrm{m} 157$, one of many MCMV-encoded proteins, can be recognized by different Ly 49 receptors, both activating and inhibitory, suggested that other mechanisms of MCMV recognition might exist in other mouse strains.

3.2. $L y 49 P / m 04 / H-2 D^{k}$ Axis. In this second mechanism of MCMV-infected cell recognition, an activating Ly49 receptor recognizes "altered" self-MHCI molecules rather than a viral MHCI homologue; this alteration of self-MHCI molecules stems from their association with viral protein. This type of recognition could be used by NK cells from MA/MyJ mice, which are resistant to MCMV even though they lack Ly49H. Indeed, MA/MyJ resistance has been associated with a genetic interaction between the NKC and the $\mathrm{H}-2^{k}$ loci or the $H-2^{k}$ locus alone [48-50]. At the cellular level, MA/MyJ resistance is correlated with the expression of the activating Ly49P receptor. This receptor can recognize infected cells given the surface expression of two specific elements: the viral m04/gp34 protein in association with $\mathrm{H}-2 \mathrm{D}^{\mathrm{k}}$ molecules (Figure 1(c)). In order to dissect the contribution of $\mathrm{H}-2$ to this recognition, chimeric $\mathrm{H}-2$ molecules were produced, in which domains were swapped between $\mathrm{H}-2 \mathrm{D}^{\mathrm{b}}$ and $\mathrm{H}-$ $2 \mathrm{D}^{\mathrm{k}}$; these chimeric molecules were used to show that the $\mathrm{H}-2 \mathrm{D}^{\mathrm{k}}$-binding platform in particular is required for this recognition. Furthermore, the ectopic expression of m04/gp34 alone cannot stimulate Ly49P-bearing reporter cells in the absence of infection. This stimulation, however, is restored when target cells are infected with a viral deletion mutant lacking $m 04(\triangle m 04 \mathrm{MCMV})$, suggesting that another host or viral factor is necessary for $\mathrm{Ly} 49 \mathrm{P} / \mathrm{m} 04 / \mathrm{H}-2 \mathrm{D}^{\mathrm{k}}$ mediated recognition of infected cells. m04/gp34 is crucial for the in vivo NK cell response in MA/MyJ mice, since 


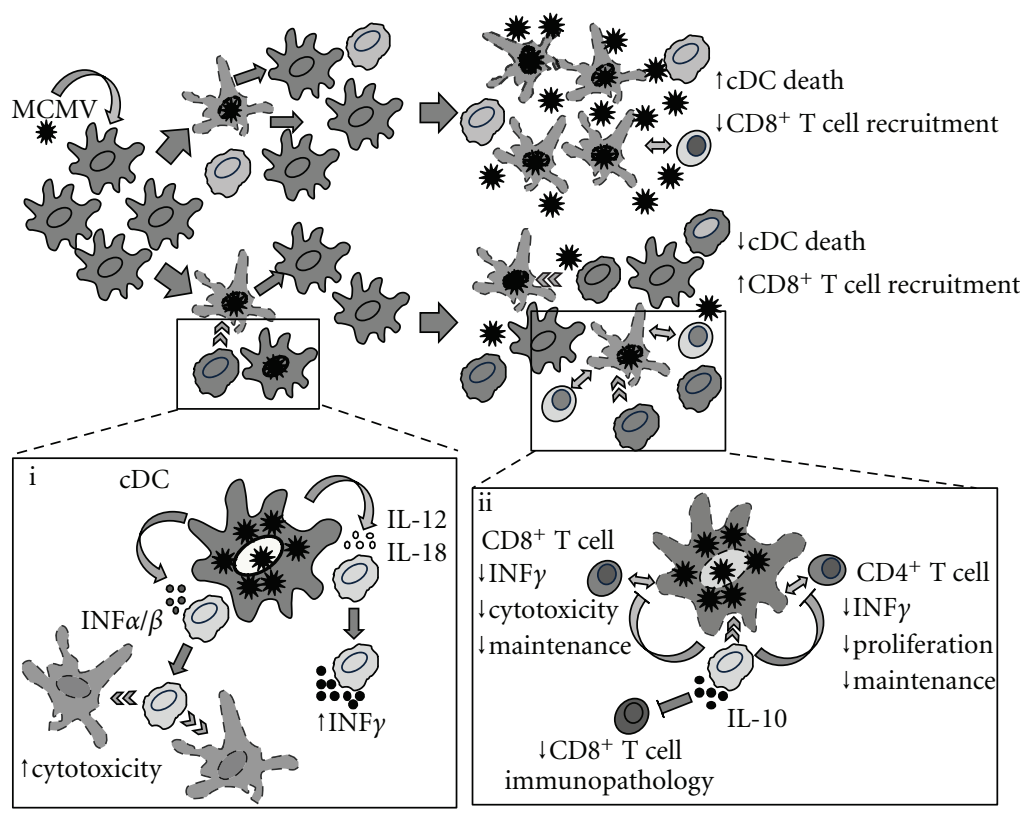

(a)

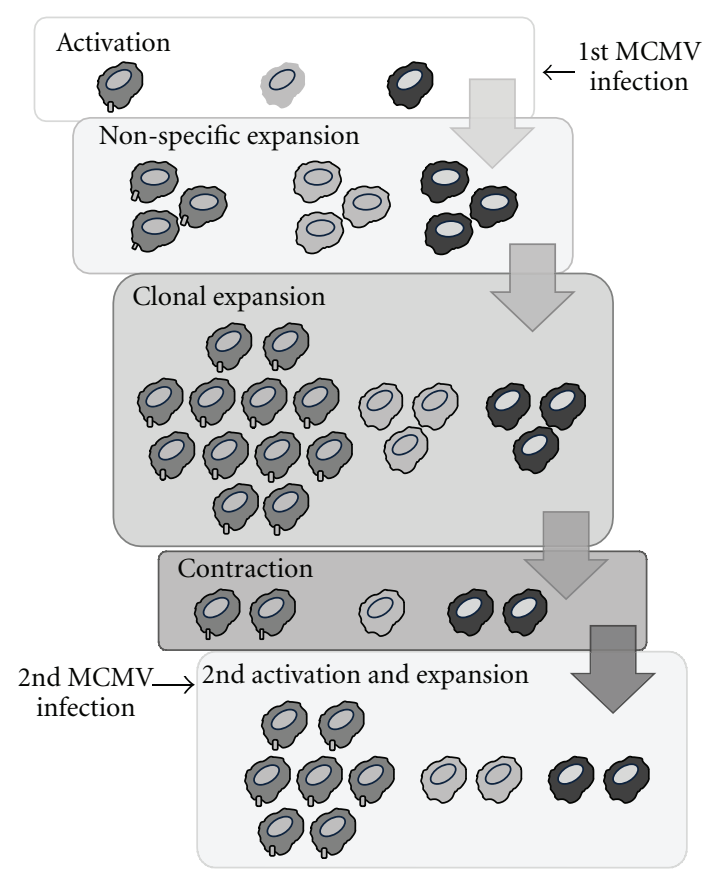

(b)

FIGURE 2: Different regulatory roles of $\mathrm{Ly}_{49 \mathrm{H}^{+}} \mathrm{NK}$ cells in adaptive immune responses. (a) $\mathrm{Ly}_{4} 9 \mathrm{H}^{+} \mathrm{NK}$ cells rapidly control viral replication and protect $\mathrm{cDCs}$ from MCMV infection. This allows for rapid recruitment and priming of $\mathrm{CD}^{+} \mathrm{T}$ cells and reduces viral and selfimmunopathology. (i) Interaction of NK cells with MCMV-infected conventional dendritic cells (cDCs) induces the former's proliferation and cytotoxicity via IFN- $\alpha / \beta$ or secretion of IFN- $\gamma$ via IL-12/IL-18. (ii) $\mathrm{Ly}_{4} 4 \mathrm{H}^{+} \mathrm{NK}$ killing of infected cDCs will also reduce the priming of $\mathrm{CD}^{+} / \mathrm{CD}^{+} \mathrm{T}$ cells and dampen the ensuing adaptive immune responses; NK cells will produce IL-10, which will limit excessive CD8 ${ }^{+} \mathrm{T}$ cell responses, thus reducing immunopathology. (b) Schematic representation of NK cell proliferative responses upon primary and secondary MCMV infections. $\uparrow$ : increase, $\downarrow$ : decrease.

normally resistant $\mathrm{MA} / \mathrm{MyJ}$ mice are unable to control viral replication following infection with $\triangle m 04 \mathrm{MCMV}$ [51] (Figure 1(d)).

The importance of $\mathrm{H}-2 \mathrm{D}^{\mathrm{k}}$ in the resistance of MA/MyJ mice was also demonstrated in vivo: (1) by the creation of an $\mathrm{H}-2^{\mathrm{k}}$ congenic mouse panel; (2) by the transgenesis of $\mathrm{H}-2 \mathrm{D}^{\mathrm{k}}$ into susceptible $\mathrm{H}-2^{\mathrm{b}}$ mice. In both cases, the mice became capable of controlling the viral infection, as opposed to $\mathrm{H}-2^{\mathrm{b}}$ animals $[50,52,53]$. Xie et al. tested their $\mathrm{H}-2^{\mathrm{k}}$ congenic mouse panel further and showed that Ly $49 \mathrm{G}^{+} \mathrm{NK}$ cells expanded preferentially in these mice at 90 hours p.i. Their resistance to MCMV infection was abrogated following depletion of Ly $49 \mathrm{G}^{+}$NK cells, which suggests that Ly49G is somehow involved $[52,53]$. Since an inhibitory receptor cannot directly mediate resistance to MCMV, the authors hypothesized that appropriate licensing via Ly49G:H-2 $D^{k}$ interaction renders NK cells functional, capable of subsequently recognizing and eliminating MCMV through Ly49P/m04/H-2D ${ }^{\mathrm{k}}$ (Figure 1(c)). Nevertheless, it remains unclear how or if licensing contributes to the ability of NK cells to resolve MCMV infection. Indeed, it has recently been shown that unlicensed NK cells respond better to MCMV infection [54]. Regardless of the role of licensing, the available evidence suggests that several Ly49 receptors might be involved in the control of MCMV infection in MA/MyJ mice.
3.3. Inhibitory Ly49/m04/MHCI Axis. The contribution of inhibitory Ly49 receptors to the NK cell-mediated control of MCMV infection has recently been evaluated. Indeed, studies using BALB mice show that the presence of Ly49 inhibitory receptors negatively impacts early MCMV resistance, that is, it makes mice susceptible to the virus [55]. BALB mice are considered susceptible, that is to say that they are unable to control the replication of WT MCMV in the spleen at day 3 p.i. However, infecting these mice with $\triangle m 04$ MCMV results in a significant reduction of viral loads in this organ and increased NK cell proliferation in a $\mathrm{H}$-2-dependent manner. Indeed, BALB mice of the $\mathrm{H}$ $2^{\mathrm{k}}$ (BALB.K) background showed the greatest reduction in viral load upon infection with $\triangle m 04 \mathrm{MCMV}$, whereas none was seen in $\mathrm{H}-2^{\mathrm{b}}$ mice (BALB.By); the viral load reduction in $\mathrm{H}-2^{\mathrm{d}}$ mice $(\mathrm{BALB} / \mathrm{c})$ was an intermediate value between that of $\mathrm{H}-2^{\mathrm{k}}$ and $\mathrm{H}-2^{\mathrm{b}}$ mice. This reduction in viral load is due to the absence of $m 04 / g p 34$, which maintains surface expression of MHCI molecules, and the action of activating receptors such as NKG2D. As such, in WT MCMV-infected cells, sufficient levels of MHCI molecules are displayed on the cell surface; this allows inhibitory Ly49 receptors to be triggered and to deliver their inhibitory signal, the result of which is the unresponsiveness of NK cells (Figure 1(e)). In the case of $\triangle m 04 \mathrm{MCMV}$ infection, the expression of MHCI molecules is severely downregulated, allowing NK 
cell activation through the "missing-self" mechanism and improved control of viral spread (Figure 1(f)). Furthermore, viral loads were shown to return to high levels following depletion of certain inhibitory Ly $49^{+}$NK cell fractions during $\triangle m 04 \mathrm{MCMV}$ infection of $\mathrm{H}-2^{\mathrm{d}}$ BALB mice. This effect was more pronounced following $\mathrm{Ly}_{4} 4 \mathrm{G}^{+} \mathrm{NK}$ cell depletion than $\mathrm{Ly}_{49 \mathrm{C}^{+}}$or Ly49A $\mathrm{A}^{+} \mathrm{NK}$ cell depletion. In summary altered self-recognition by activating Ly49 receptors, requiring the presence of self-MHCI molecules associated with viral products, implicates involvement of inhibitory Ly49 receptors, which depending on mice strain can have opposite effects. Thus, when the predominant signal is m04-dependent activation (e.g., MA/MyJ via Ly49P), m04 ablation allows enhanced viral replication; however, when the predominant signal is m04-dependent inhibition (e.g., BALB via Ly49G/A), absence of $m 04$ allows enhanced control of virus replication.

In short, these findings suggest that the early, NK celldependent control of viral replication is not solely due to activating Ly49 receptors, but rather that it involves a delicate interplay between signals generated by both activating and inhibitory receptors.

\section{Beyond Natural Killing: Regulation of Inflammatory and Adaptive Responses by NK Cells}

NK cells have classically been thought of as effectors of the antiviral response through their ability to directly eliminate infected cells. However, recent evidence demonstrates that their role extends beyond that of effector lymphocytes into regulators of the inflammatory and adaptive immune response against viral infection. More specifically, $\mathrm{Ly}_{49 \mathrm{H}^{+}}$ NK cells are essential for the protection and preservation of cDCs, which can themselves stimulate NK cells and potentiate their response [56, 57]. Nevertheless, infection does occur; NK cell-dependent elimination of cDCs and, in particular, macrophages, reduces the secretion of TNF- $\alpha$, a major contributor to hemophagocytic lymphohistiocytosis syndrome during MCMV infection [26]. In addition, Ly49Hm157-dependent reduction of viral burden decreases the duration and intensity of pDC activation, which limits very high systemic levels of type I IFN and other cytokines that can be detrimental to the host $[32,58-60]$. In doing so, NK cells also accelerate the recruitment and expansion of antiMCMV specific $\mathrm{CD}^{+} \mathrm{T}$ cells, as it was shown that in mice devoid of Ly49H, the appearance of $\mathrm{CD}^{+} \mathrm{T}$ cells is delayed by $\sim 1$ day (Figure 2) [57].

Triggering by $\mathrm{m} 157$ is essential for $\mathrm{Ly}_{4} 9 \mathrm{H}^{+} \mathrm{NK}$ cell amplification and maintenance. In the context of MCMV infection, signaling through Ly49H/DAP12 induces NK cell clonal proliferation, ultimately leading to an increase in the Ly $49 \mathrm{H}^{+} \mathrm{NK}$ cell frequency from $50 \%$ to $80 \%$ and clearance of the virus in the spleen and liver by day 6 p.i. [32]. Conversely, in $\mathrm{Ly} 49 \mathrm{H}^{-/-}$mice, the overall amount of NK cells declines past uninfected levels, leading to high viral titers in the spleen at the same time point [59]. Another study, by Lee et al., attempted to dissect the role of Ly49H in various aspect of the $\mathrm{NK}$ cell response using $L y 49 \mathrm{~h}$ or perforin 1 (Prf1) knockout mice. For instance, Ly49Hexpressing $\mathrm{Prfl}^{-/-}$mice were susceptible to MCMV infection, even though $\mathrm{Ly} 49 \mathrm{H}^{+} \mathrm{NK}$ cells proliferated substantially. Although in these mice the NK cytotoxic function was absent, they survived a dose that killed $L y 49 \mathrm{~h}^{-/-} \mathrm{Prfl}^{-/-}$ double-knockout mice. Therefore, Ly49H expression was protective. This was explained by the fact that $\mathrm{Ly} 49 \mathrm{H}^{+} \mathrm{NK}$ cells secreted IL-10, a potent immunosuppressive cytokine capable of limiting $\mathrm{CD} 8^{+} \mathrm{T}$ cell cytotoxicity as well as IFN$\gamma$ and TNF- $\alpha$ secretion (Figure 2(a)ii) [59]. Thus, Ly49H is required for the maintenance of NK cells until late timepoints after infection, allowing them to exert a regulatory function over $\mathrm{CD}^{+} \mathrm{T}$ cells and the immunopathology they can cause.

Paradoxically, the presence of activating Ly49 receptors can also be detrimental to the subsequent adaptive immune response by promoting viral persistence. For example, while Ly $49 \mathrm{H}^{+}$mice could clear infected cDCs better than their Ly $49 \mathrm{H}^{-}$counterparts, killing of these antigen-presenting cells (APCs) prevented them from priming naïve, virusspecific $\mathrm{T}$ cells [61]. This lack of appropriate T-cell priming in $\mathrm{Ly} 49 \mathrm{H}^{+}$mice resulted in the reduced activation, cytotoxicity, IFN- $\gamma$ secretion, and maintenance of $\mathrm{CD} 8^{+} \mathrm{T}$ cells, as well as the reduced IFN- $\gamma$ secretion and maintenance of $\mathrm{CD} 4^{+}$ $\mathrm{T}$ cell, contributing to MCMV persistence in the salivary glands (Figure 2(a)ii). However, MCMV-infected, immature cDCs have also been shown to induce anergy, poor effector functions, and inferior recall responses in $\mathrm{CD} 4^{+}$and $\mathrm{CD} 8^{+} \mathrm{T}$ cells [62]. Further research is needed to determine the precise effect of $\mathrm{cDC}$ clearance in the overall response to the virus. Nevertheless, $\mathrm{Ly} 49 \mathrm{H}^{+}$NK cell recognition and removal of MCMV-infected cells can unquestionably shape the course of the adaptive immune response.

\section{Memory-Like NK Cells}

Another unforeseen NK cell role was evidenced by the discovery that, following activation, NK cells acquire features seen in memory T-cell populations. The existence of memory-like NK cells was first hinted at in a study of chemically-induced contact hypersensitivity (CHS). In this model, mice lacking $\mathrm{T}$ and $\mathrm{B}$ lymphocytes, the classical mediators of CHS, remained able to elicit an inflammatory response through hepatic NK cells, as measured by dermatitis [63]. Sun et al. [64] followed up on this observation using an MCMV model of infection and adoptive transfer into DAP $12^{-/-}$mice. The investigators noticed that only Ly $49 \mathrm{H}^{+}$ NK cells underwent specific clonal expansion before contraction and persisted for up to 70 days p.i. This was rather astonishing as the generally accepted half-life of mature NK cells is 7 to 17 days [65]. Importantly, irrespective of initial transfer numbers the frequency of the MCMV-specific NKcell response was similar to primary T-cells responses. They also observed that these long-lived, memory-like NK cells were $\mathrm{m} 157$-specific and had superior effector responses upon rechallenge with MCMV as compared to naïve NK cells [64]. 
The existence of memory-like NK cells was also confirmed by transferring cytokine-activated NK cells into naïve hosts and observing that they could still be detected as far as 22 days after transfer [66]. These memory NK cells were found to have similar cytotoxic activity to that of their naïve counterparts. Moreover, they were unable to constitutively produce IFN- $\gamma$ yet upon restimulation produced significantly more of this cytokine than naïve NK cells. More recently, again in the CHS model, virusspecific memory-like NK cells were generated in response to influenza virus, VSV or HSV-1 infection. In this case, the adoptive transfer of specific, virus-sensitized hepatic NK cells into naive mice improved their survival following infection with a lethal dose of virus; this was dependent on the CXCR6 chemokine receptor expressed on NK cells [67].

All of these experiments have shown that innate immune cells can retain an intrinsic memory of prior activation, a function until now restricted to antigen-specific, adaptive immune lymphocytes. However, most of the aforementioned studies were performed with immunodeficient donor and recipient mice. Given the adaptability of the immune system, it is possible that donor NK cells, once transferred into these recipients, may have taken up more responsibility (i.e., memory status) than they normally would in WT animals in order to compensate for the absence of $\mathrm{T}$ and $\mathrm{B}$ cells. As for the work performed by Sun et al., even though their donor NK cells came from WT mice, they were transferred into neonate animals, whose adaptive immune responses are arguably as ineffective as that of immunodeficient mice.

\section{Conclusions}

Since their discovery over 30 years ago, it has become clear that NK cells possess numerous functions, going beyond their original "natural killers of tumors" role. Among these, they act as a first line of defense in the context of infection with a variety of pathogens, in particular viruses. Humans have been coevolving for millions of years with some of these viruses, namely CMVs; therefore, they "know us better than we know ourselves", having shaped our immune system and been shaped in return.

Mouse models of MCMV infection revealed that NK cells are not merely "killers" of infected targets, but very complex lymphocytes endowed with the molecular machinery necessary to perform a broad spectrum of functions. As part of the innate system, NK cells can immediately react to an infectious insult via fixed, germ-line encoded receptors that recognize pathogen-associated motifs. As the infection progresses, NK cells adapt, modulating the level of inflammatory mediators produced during the initial steps of infection. Finally, as adaptive responders, NK cells show clonal expansion, maintenance, and memory of previous insults in the case of reinfection.

Nevertheless, several questions remain: do NK cells recognize infections other than MCMV with their Ly49 repertoire of receptors? Can triggering of other NK receptors by MCMV-infected cells induce the same NK cell outcomes as in the Ly49H-m157 model (e.g., clonal expansion, memory, etc.)? What of other pathogens or different host species? Why does the host express so many different NK receptors targeting MCVM, several with opposite effects on NK cell activity (i.e., inhibitory versus activating)? Does the host benefit from the infection? If so, how? The possible paths of investigation are plentiful. As more inquiries are resolved, which is currently the case in the thriving field of NK cells in cancer, we will be able to harness the power of these lymphocytes in the treatment of infectious diseases $[68,69]$.

\section{Acknowledgments}

The authors apologize to their colleagues whose work was not cited due to space limitations. Research in our laboratory is supported by grants of the Canadian Institutes of Health Research (CIHR). S. M. Vidal is the recipient of a Canada Research Chair; M. Pyzik is supported by a CIHR Doctoral Award.

\section{References}

[1] S. D. Burke, V. F. Barrette, J. Gravel et al., "Uterine NK cells, spiral artery modification and the regulation of blood pressure during mouse pregnancy," American Journal of Reproductive Immunology, vol. 63, no. 6, pp. 472-481, 2010.

[2] L. L. Lanier, "Evolutionary struggles between NK cells and viruses," Nature Reviews Immunology, vol. 8, no. 4, pp. 259$268,2008$.

[3] C. A. Biron, K. S. Byron, and J. L. Sullivan, "Severe herpesvirus infections in an adolescent without natural killer cells," New England Journal of Medicine, vol. 320, no. 26, pp. 1731-1735, 1989.

[4] R. Gazit, B. Z. Garty, Y. Monselise et al., "Expression of KIR2DL1 on the entire NK cell population: a possible novel immunodeficiency syndrome," Blood, vol. 103, no. 5, pp. 1965-1966, 2004.

[5] L. L. Lanier, "Up on the tightrope: natural killer cell activation and inhibition," Nature Immunology, vol. 9, no. 5, pp. 495-502, 2008.

[6] T. Crough and R. Khanna, "Immunobiology of human cytomegalovirus: from bench to bedside," Clinical Microbiology Reviews, vol. 22, pp. 76-98, 2009.

[7] V. J. Lisnić, A. Krmpotić, and S. Jonjić, "Modulation of natural killer cell activity by viruses," Current Opinion in Microbiology, vol. 13, pp. 530-539, 2010.

[8] M. G. Brown and A. A. Scalzo, "NK gene complex dynamics and selection for NK cell receptors," Seminars in Immunology, vol. 20, no. 6, pp. 361-368, 2008.

[9] R. Biassoni, "Human natural killer receptors, co-receptors, and their ligands," Current Protocols in Immunology, no. 84, pp. 14.10.1-14.10.40, 2009.

[10] P. Höglund and P. Brodin, "Current perspectives of natural killer cell education by MHC class i molecules," Nature Reviews Immunology, vol. 10, no. 10, pp. 724-734, 2010.

[11] K. M. Hsu, J. R. Pratt, W. J. Akers, S. I. Achilefu, and W. M. Yokoyama, "Murine cytomegalovirus displays selective infection of cells within hours after systemic administration," Journal of General Virology, vol. 90, no. 1, pp. 33-43, 2009.

[12] T. Sacher, J. Podlech, C. A. Mohr et al., "The major virusproducing cell type during murine cytomegalovirus infection, 
the hepatocyte, is not the source of virus dissemination in the host," Cell Host and Microbe, vol. 3, no. 4, pp. 263-272, 2008.

[13] T. A. Banks, S. Rickert, C. A. Benedict et al., "A lymphotoxinIFN- $\beta$ axis essential for lymphocyte survival revealed during cytomegalovirus infection," Journal of Immunology, vol. 174, no. 11, pp. 7217-7225, 2005.

[14] K. Schneider, A. Loewendorf, C. De Trez et al., "Lymphotoxinmediated crosstalk between B cells and splenic stroma promotes the initial type I interferon response to cytomegalovirus," Cell Host and Microbe, vol. 3, no. 2, pp. 67-76, 2008.

[15] K. Tabeta, P. Georgel, E. Janssen, X. Du, K. Hoebe et al., "Toll-like receptors 9 and 3 as essential components of innate immune defense against mouse cytomegalovirus infection," Proceedings of the National Academy of Sciences of the United States of America, vol. 101, pp. 3516-3521, 2004.

[16] A. Krug, A. R. French, W. Barchet et al., "TLR9-dependent recognition of MCMV by IPC and DC generates coordinated cytokine responses that activate antiviral NK cell function," Immunity, vol. 21, no. 1, pp. 107-119, 2004.

[17] K. Tabeta, K. Hoebe, E. M. Janssen et al., "The Unc93b1 mutation $3 \mathrm{~d}$ disrupts exogenous antigen presentation and signaling via Toll-like receptors 3, 7 and 9," Nature Immunology, vol. 7, no. 2, pp. 156-164, 2006.

[18] V. R. DeFilippis, D. Alvarado, T. Sali, S. Rothenburg, and K. Früh, "Human cytomegalovirus induces the interferon response via the DNA sensor ZBP1," Journal of Virology, vol. 84, no. 1, pp. 565-598, 2010.

[19] V. A. K. Rathinam, Z. Jiang, S. N. Waggoner et al., "The AIM2 inflammasome is essential for host defense against cytosolic bacteria and DNA viruses," Nature Immunology, vol. 11, no. 5, pp. 395-402, 2010.

[20] M. Swiecki, S. Gilfillan, W. Vermi, Y. Wang, and M. Colonna, "Plasmacytoid dendritic cell ablation impacts early interferon responses and antiviral NK and CD8+ T cell accrual," Immunity, vol. 33, no. 6, pp. 955-966, 2010.

[21] G. C. Pien, A. R. Satoskar, K. Takeda, S. Akira, and C. A. Biron, "Cutting edge: selective IL-18 requirements for induction of compartmental IFN- $\gamma$ responses during viral infection," Journal of Immunology, vol. 165, no. 9, pp. 4787-4791, 2000.

[22] K. B. Nguyen, T. P. Salazar-Mather, M. Y. Dalod et al., "Coordinated and distinct roles for IFN- $\alpha \beta$, IL-12, and IL-15 regulation of NK cell responses to viral infection," Journal of Immunology, vol. 169, no. 8, pp. 4279-4287, 2002.

[23] D. M. Andrews, A. A. Scalzo, W. M. Yokoyama, M. J. Smyth, and M. A. Degli-Esposti, "Functional interactions between dendritic cells and NK cells during viral infection," Nature Immunology, vol. 4, no. 2, pp. 175-181, 2003.

[24] T. P. Salazar-Mather, J. S. Orange, and C. A. Biron, "Early murine cytomegalovirus (MCMV) infection induces liver natural killer $(\mathrm{NK})$ cell inflammation and protection through macrophage inflammatory protein $1 \alpha$ (MIP- $1 \alpha)$-dependent pathways," Journal of Experimental Medicine, vol. 187, no. 1, pp. 1-14, 1998.

[25] N. Sumaria, S. L. H. Van Dommelen, C. E. Andoniou, M. J. Smyth, A. A. Scalzo, and M. A. Degli-Esposti, "The roles of interferon $-\gamma$ and perforin in antiviral immunity in mice that differ in genetically determined NK-cell-mediated antiviral activity," Immunology and Cell Biology, vol. 87, no. 7, pp. 559566, 2009.

[26] S. L. H. van Dommelen, N. Sumaria, R. D. Schreiber, A. A. Scalzo, M. J. Smyth, and M. A. Degli-Esposti, "Perforin and granzymes have distinct roles in defensive immunity and immunopathology," Immunity, vol. 25, no. 5, pp. 835-848, 2006.

[27] A. A. Scalzo, N. A. Fitzgerald, A. Simmons, A. B. La Vista, and G. R. Shellam, "Cmv-1, a genetic locus that controls murine cytomegalovirus replication in the spleen," Journal of Experimental Medicine, vol. 171, no. 5, pp. 1469-1483, 1990.

[28] M. Rodriguez, P. Sebastian, P. Clark, and M. G. Brown, "Cmv1-independent antiviral role of NK cells revealed in murine cytomegalovirus-infected New Zealand white mice," Journal of Immunology, vol. 173, no. 10, pp. 6312-6318, 2004.

[29] S. G. Adam, A. Caraux, N. Fodil-Cornu et al., "Cmv4, a new locus linked to the NK cell gene complex, controls innate resistance to cytomegalovirus in wild-derived mice," Journal of Immunology, vol. 176, no. 9, pp. 5478-5485, 2006.

[30] K. A. Daniels, G. Devora, W. C. Lai, C. L. O’Donnell, M. Bennett, and R. M. Welsh, "Murine cytomegalovirus is regulated by a discrete subset of natural killer cells reactive with monoclonal antibody to Ly49H," Journal of Experimental Medicine, vol. 194, no. 1, pp. 29-44, 2001.

[31] S. H. Lee, J. R. Webb, and S. M. Vidal, "Innate immunity to cytomegalovirus: the Cmv1 locus and its role in natural killer cell function," Microbes and Infection, vol. 4, no. 15, pp. 14911503, 2002.

[32] A. O. Dokun, S. Kim, H. R. C. Smith, H. S. P. Kang, D. T. Chu, and W. M. Yokoyama, "Specific and nonspecific NK cell activation during virus infection," Nature Immunology, vol. 2, no. 10, pp. 951-956, 2001.

[33] H. Arase, E. S. Mocarski, A. E. Campbell, A. B. Hill, and L. L. Lanier, "Direct recognition of cytomegalovirus by activating and inhibitory NK cell receptors," Science, vol. 296, no. 5571, pp. 1323-1326, 2002.

[34] H. R. C. Smith, J. W. Heusel, I. K. Mehta et al., "Recognition of a virus-encoded ligand by a natural killer cell activation receptor," Proceedings of the National Academy of Sciences of the United States of America, vol. 99, no. 13, pp. 8826-8831, 2002.

[35] E. J. Adams, Z. S. Juo, R. T. Venook et al., "Structural elucidation of the m157 mouse cytomegalovirus ligand for Ly49 natural killer cell receptors," Proceedings of the National Academy of Sciences of the United States of America, vol. 104, no. 24, pp. 10128-10133, 2007.

[36] I. Bubić, M. Wagner, A. Krmpotić et al., "Gain of virulence caused by loss of a gene in murine cytomegalovirus," Journal of Virology, vol. 78, no. 14, pp. 7536-7544, 2004.

[37] H. Sjölin, E. Tomasello, M. Mousavi-Jazi et al., "Pivotal role of KARAP/DAP12 adaptor molecule in the natural killer cellmediated resistance to murine cytomegalovirus infection," Journal of Experimental Medicine, vol. 195, no. 7, pp. 825-834, 2002.

[38] T. P. Cheng, A. R. French, B. F. M. Plougastel et al., "Ly49h is necessary for genetic resistance to murine cytomegalovirus," Immunogenetics, vol. 60, no. 10, pp. 565-573, 2008.

[39] N. Fodil-Cornu, S. H. Lee, S. Belanger et al., "Ly49h-deficient C57BL/6 mice: a new mouse cytomegalovirus-susceptible model remains resistant to unrelated pathogens controlled by the NK gene complex," Journal of Immunology, vol. 181, no. 9, pp. 6394-6405, 2008.

[40] S. H. Lee, A. Zafer, Y. De Repentigny et al., "Transgenic expression of the activating natural killer receptor $\mathrm{Ly} 49 \mathrm{H}$ confers resistance to cytomegalovirus in genetically susceptible mice," Journal of Experimental Medicine, vol. 197, no. 4, pp. 515-526, 2003.

[41] A. Kielczewska, H. S. Kim, L. L. Lanier, N. Dimasi, and S. M. Vidal, "Critical residues at the Ly49 natural killer receptor's 
homodimer interface determine functional recognition of m157, a mouse cytomegalovirus MHC class I-like protein," Journal of Immunology, vol. 178, no. 1, pp. 369-377, 2007.

[42] A. H. Davis, N. V. Guseva, B. L. Ball, and J. W. Heusel, "Characterization of murine cytomegalovirus ml57 from infected cells and identification of critical residues mediating recognition by the NK cell receptor Ly49H," Journal of Immunology, vol. 181, no. 1, pp. 265-275, 2008.

[43] N. V. Guseva, C. A. Fullenkamp, P. W. Naumann et al., "Glycosylation contributes to variability in expression of murine cytomegalovirus $\mathrm{m} 157$ and enhances stability of interaction with the NK-cell receptor Ly49H," European Journal of Immunology, vol. 40, no. 9, pp. 2618-2631, 2010.

[44] D. W. McVicar, R. Winkler-Pickett, L. S. Taylor et al., "Aberrant DAP12 signaling in the 129 strain of mice: implications for the analysis of gene-targeted mice," Journal of Immunology, vol. 169, no. 4, pp. 1721-1728, 2002.

[45] L. L. Lanier, "NK cell recognition," Annual Review of Immunology, vol. 23, pp. 225-274, 2005.

[46] A. J. Corbett, J. D. Coudert, C. A. Forbes, and A. A. Scalzo, "Functional consequences of natural sequence variation of murine cytomegalovirus $\mathrm{m} 157$ for Ly49 receptor specificity and NK cell activation," Journal of Immunology, vol. 186, no. 3, pp. 1713-1722, 2011.

[47] V. Voigt, C. A. Forbes, J. N. Tonkin et al., "Murine cytomegalovirus m157 mutation and variation leads to immune evasion of natural killer cells," Proceedings of the National Academy of Sciences of the United States of America, vol. 100, no. 23, pp. 13483-13488, 2003.

[48] M.-P. Desrosiers, A. Kielczewska, J.-C. Loredo-Osti et al., "Epistasis between mouse Klra and major histocompatibility complex class I loci is associated with a new mechanism of natural killer cell-mediated innate resistance to cytomegalovirus infection," Nature Genetics, vol. 37, no. 6, pp. 593-599, 2005.

[49] A. Dighe, M. Rodriguez, P. Sabastian, X. Xie, M. McVoy, and M. G. Brown, "Requisite H2k role in NK cell-mediated resistance in acute murine cytomegalovirus-infected MA/My mice," Journal of Immunology, vol. 175, no. 10, pp. 6820-6828, 2005.

[50] X. Xie, A. Dighe, P. Clark, P. Sabastian, S. Buss, and M. G. Brown, "Deficient major histocompatibility complex-linked innate murine cytomegalovirus immunity in MA/My.L-H2 mice and viral downregulation of H-2 class I proteins," Journal of Virology, vol. 81, no. 1, pp. 229-236, 2007.

[51] A. Kielczewska, M. Pyzik, T. Sun et al., "Ly49P recognition of cytomegalovirus-infected cells expressing H2-D and CMVencoded m04 correlates with the NK cell antiviral response," Journal of Experimental Medicine, vol. 206, no. 3, pp. 515-523, 2009.

[52] X. Xie, M. D. Stadnisky, and M. G. Brown, "MHC class I D locus and Ly49G2+ NK cells confer $\mathrm{H}-2$ resistance to murine cytomegalovirus," Journal of Immunology, vol. 182, no. 11, pp. 7163-7171, 2009.

[53] X. Xie, M. D. Stadnisky, E. R. Coats et al., "MHC class I D expression in hematopoietic and nonhematopoietic cells confers natural killer cell resistance to murine cytomegalovirus," Proceedings of the National Academy of Sciences of the United States of America, vol. 107, no. 19, pp. 8754-8759, 2010.

[54] M. T. Orr, W. J. Murphy, and L. L. Lanier, “"Unlicensed” natural killer cells dominate the response to cytomegalovirus infection," Nature Immunology, vol. 11, no. 4, pp. 321-327, 2010.
[55] M. Babić, M. Pyzik, B. Zafirova et al., "Cytomegalovirus immunoevasin reveals the physiological role of "missing self" recognition in natural killer cell dependent virus control in vivo," Journal of Experimental Medicine, vol. 207, no. 12, pp. 2663-2673, 2010.

[56] C. E. Andoniou, S. L. H. van Dommelen, V. Voigt et al., "Interaction between conventional dendritic cells and natural killer cells is integral to the activation of effective antiviral immunity," Nature Immunology, vol. 6, no. 10, pp. 1011-1019, 2005.

[57] S. H. Robbins, G. Bessou, A. Cornillon et al., "Natural killer cells promote early CD8 $\mathrm{T}$ cell responses against cytomegalovirus.," PLoS Pathogens, vol. 3, no. 8, article e123, 2007.

[58] S. H. Lee, S. Girard, D. Macina et al., "Susceptibility to mouse cytomegalovirus is associated with deletion of an activating natural killer cell receptor of the C-type lectin superfamily," Nature Genetics, vol. 28, no. 1, pp. 42-45, 2001.

[59] S. H. Lee, K. S. Kim, N. Fodil-Cornu, S. M. Vidal, and C. A. Biron, "Activating receptors promote NK cell expansion for maintenance, IL-10 production, and CD8 $\mathrm{T}$ cell regulation during viral infection," Journal of Experimental Medicine, vol. 206, no. 10, pp. 2235-2251, 2009.

[60] V. Bekiaris, O. Timoshenko, T. Z. Hou et al., "Ly49H+ NK cells migrate to and protect splenic white pulp stroma from murine cytomegalovirus infection," Journal of Immunology, vol. 180, no. 10, pp. 6768-6776, 2008.

[61] D. M. Andrews, M. J. Estcourt, C. E. Andoniou et al., "Innate immunity defines the capacity of antiviral $\mathrm{T}$ cells to limit persistent infection," Journal of Experimental Medicine, vol. 207, no. 6, pp. 1333-1343, 2010.

[62] C. A. Benedict, A. Loewendorf, Z. Garcia, B. R. Blazar, and E. M. Janssen, "Dendritic cell programming by cytomegalovirus stunts naive $\mathrm{T}$ cell responses via the $\mathrm{PD}-\mathrm{L} 1 / \mathrm{PD}-1$ pathway," Journal of Immunology, vol. 180, no. 7, pp. 4836-4847, 2008.

[63] J. G. O’Leary, M. Goodarzi, D. L. Drayton, and U. H. von Andrian, "T cell- and B cell-independent adaptive immunity mediated by natural killer cells," Nature Immunology, vol. 7, no. 5, pp. 507-516, 2006.

[64] J. C. Sun, J. N. Beilke, and L. L. Lanier, "Adaptive immune features of natural killer cells," Nature, vol. 457, no. 7229, pp. 557-561, 2009.

[65] A. M. Jamieson, P. Isnard, J. R. Dorfman, M. C. Coles, and D. H. Raulet, "Turnover and proliferation of NK cells in steady state and lymphopenic conditions," Journal of Immunology, vol. 172, no. 2, pp. 864-870, 2004.

[66] M. A. Cooper, J. M. Elliott, P. A. Keyel, L. Yang, J. A. Carrero, and W. M. Yokoyama, "Cytokine-induced memorylike natural killer cells," Proceedings of the National Academy of Sciences of the United States of America, vol. 106, no. 6, pp. 1915-1919, 2009.

[67] S. Paust, H. S. Gill, B.-Z. Wang et al., "Critical role for the chemokine receptor CXCR6 in NK cell-mediated antigenspecific memory of haptens and viruses," Nature Immunology, vol. 11, no. 12, pp. 1127-1135, 2010.

[68] M. Terme, E. Ullrich, N. F. Delahaye, N. Chaput, and L. Zitvogel, "Natural killer cell-directed therapies: moving from unexpected results to successful strategies," Nature Immunology, vol. 9, no. 5, pp. 486-494, 2008.

[69] S. K. Lee and S. Gasser, "The role of natural killer cells in cancer therapy," Frontiers in Bioscience, vol. 2, pp. 380-391, 2010. 


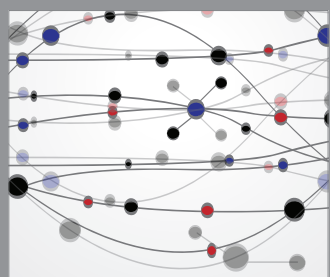

The Scientific World Journal
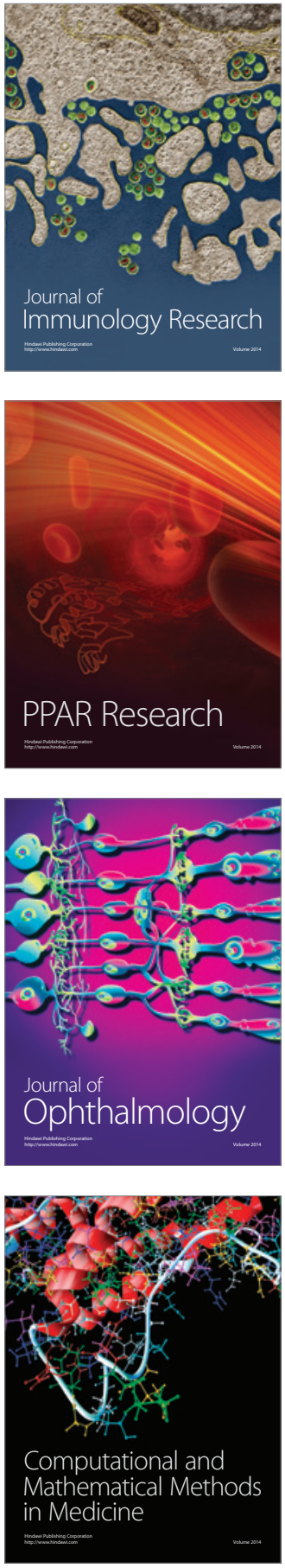

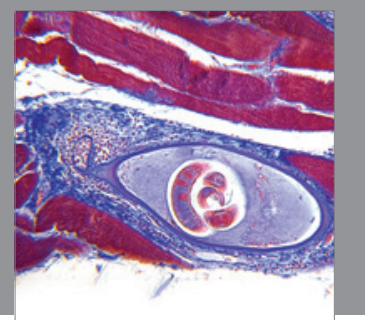

Gastroenterology

Research and Practice
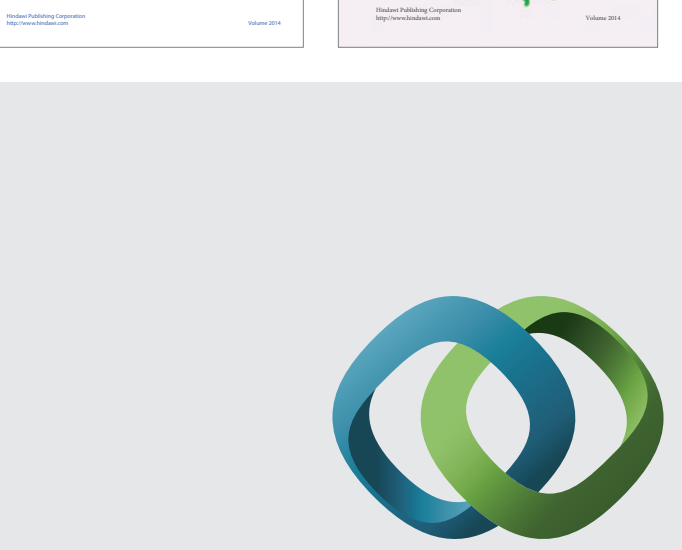

\section{Hindawi}

Submit your manuscripts at

http://www.hindawi.com
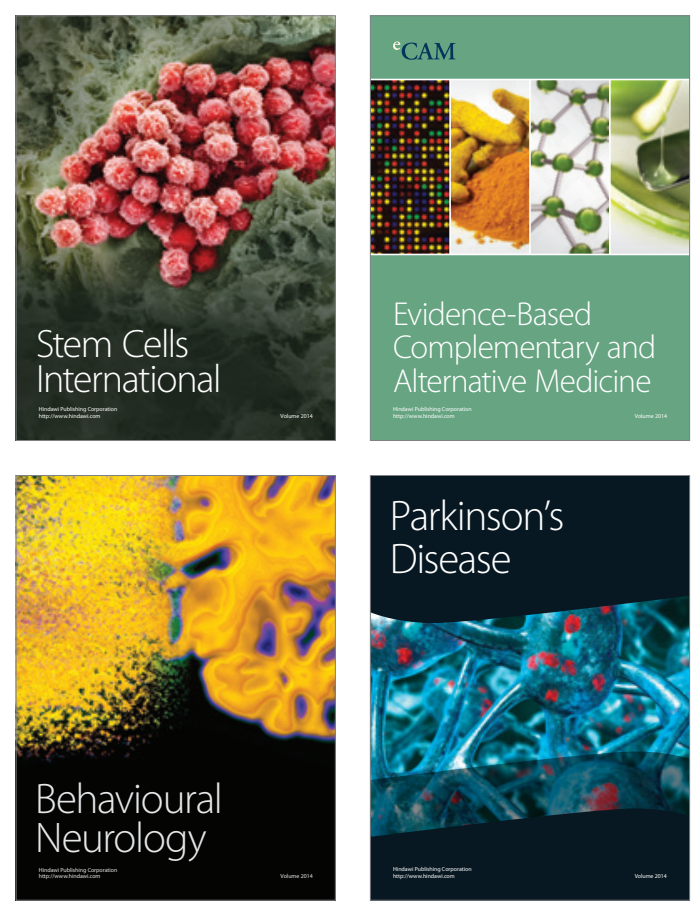

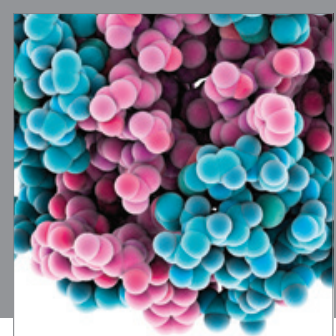

Journal of
Diabetes Research

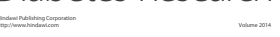

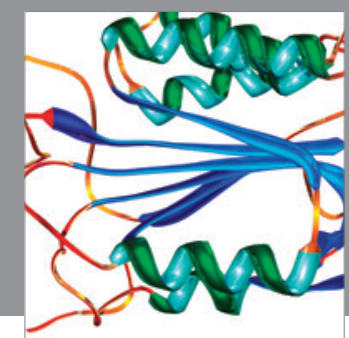

Disease Markers
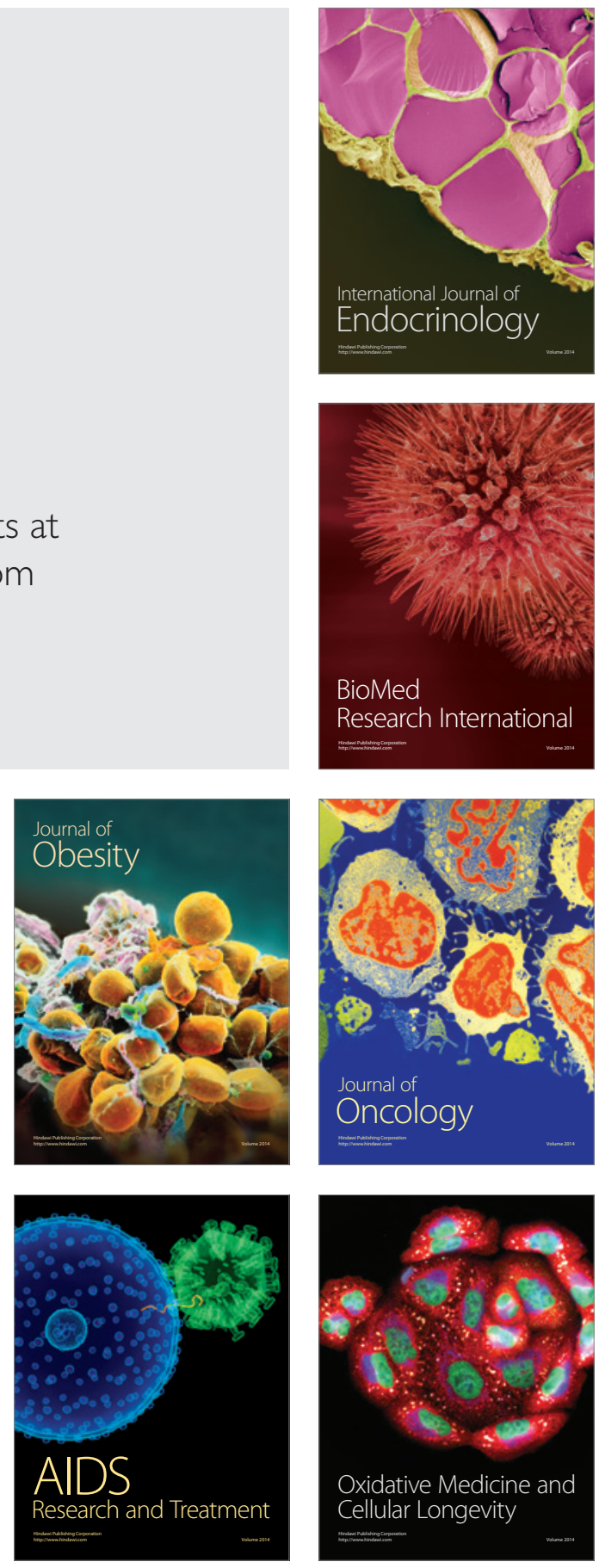